\title{
Sex and gender in interstitial lung diseases
}

\author{
Leticia Kawano-Dourado ${ }^{1,2,3}$, Marilyn K. Glassberg ${ }^{4}$, Deborah Assayag ${ }^{5}$, Raphaël Borie $\mathbb{1}^{6}$ and \\ Kerri A. Johannson (10),8
}

\author{
Number 4 in the Series "Sex and gender in lung disease" \\ Edited by Jason Weatherald, Marc Humbert and Renata Riha
}

\begin{abstract}
${ }^{1}$ HCor Research Institute, Hospital do Coracao, Sao Paulo, Brazil. ${ }^{2}$ Pulmonary Division, Heart Institute (InCor), University of Sao Paulo, Sao Paulo, Brazil. ${ }^{3}$ INSERM 1152, University of Paris, Paris, France. ${ }^{4}$ Pulmonary, Critical Care, and Sleep Medicine Division, Dept of Medicine, University of Arizona College of Medicine, Phoenix, AZ, USA. ${ }^{5}$ Dept of Medicine, McGill University, Montreal, QC, Canada. ${ }^{6}$ Pulmonary Division, Hospital Bichat, Assistance Publique - Hôpitaux de Paris (AP-HP), Paris, France. ${ }^{7}$ Depts of Medicine and Community Health Sciences, University of Calgary, Calgary, AB, Canada. ${ }^{8}$ Calvin, Phoebe and Joan Snyder Institute for Chronic Diseases, University of Calgary, Calgary, AB, Canada.
\end{abstract}

Corresponding author: Kerri A. Johannson (kerri.johannson@ahs.ca)

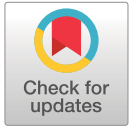

Copyright @The authors 2021

This version is distributed under the terms of the Creative Commons Attribution Non-Commercial Licence 4.0. For commercial reproduction rights and permissions contact permissions@ersnet.org

Received: 20 April 2021 Accepted: 1 July 2021

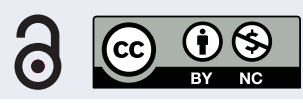

Shareable abstract (@ERSpublications)

The interplay between biological sex and behavioural gender explains a significant proportion of the variability seen in ILD. For a more individualised, patient-centred medicine, sex and gender influences in disease need to be accounted for. https://bit.ly/3hD4006

Cite this article as: Kawano-Dourado L, Glassberg MK, Assayag D, et al. Sex and gender in interstitial lung diseases. Eur Respir Rev 2021; 30: 210105 [DOI: 10.1183/16000617.0105-2021].

\section{Abstract}

Sex and gender differences influence key domains of research, lung health, healthcare access and healthcare delivery. In interstitial lung diseases (ILDs), mouse models of pulmonary fibrosis are clearly influenced by sex hormones. Additionally, short telomeres, a biomarker of telomere regulation gene mutations, are impacted by sex, while heritability unexplained by genetic variation may be attributable to gendered environmental factors that drive epigenetic control. Diseases like idiopathic pulmonary fibrosis, hypersensitivity pneumonitis, occupational ILDs, connective tissue-associated ILDs and lymphangioleiomyomatosis have different prevalence and prognosis between men and women. These differences arise from a complex interplay between biological sex and sociocultural gender influencing genetics, epigenomic modifiers, hormones, immune function, response to treatment and interaction with healthcare systems. Much work remains to be done to systematically integrate sex and gender analysis into relevant domains of science and clinical care in ILD, from strategic considerations for establishing research priorities to guidelines for establishing best clinical practices. Accounting for sex and gender in ILD is essential to the practice of individualised, patient-centred medicine.

\section{Introduction}

Sex and gender significantly impact risk, diagnosis, treatment and outcomes across several different diseases, and attention must be directed to their inclusion in research endeavours and assessment in healthcare utilisation [1]. Biological sex is expressed physiologically by gonadal and nongonadal regulation of sex differences, while gender comprises the sociocultural, environmental and behavioural variables and choices that influence self-identity, different exposure risks and healthcare utilisation [2, 3]. The influence of sex and gender is as complex and versatile as individuals and diseases themselves.

Inherent sex differences in human lung structure and function are noted in utero and manifest throughout life [2], while gender identity forms later in development and influences healthcare utilisation and differences in healthcare delivery $[2,4]$. The influence and interaction of sex and gender are therefore independent modifiers of health, disease and healthcare utilisation [3]. Sex and/or gender differences impact genetics, epigenomics, hormones, immune function, vascular health, response to treatments and 


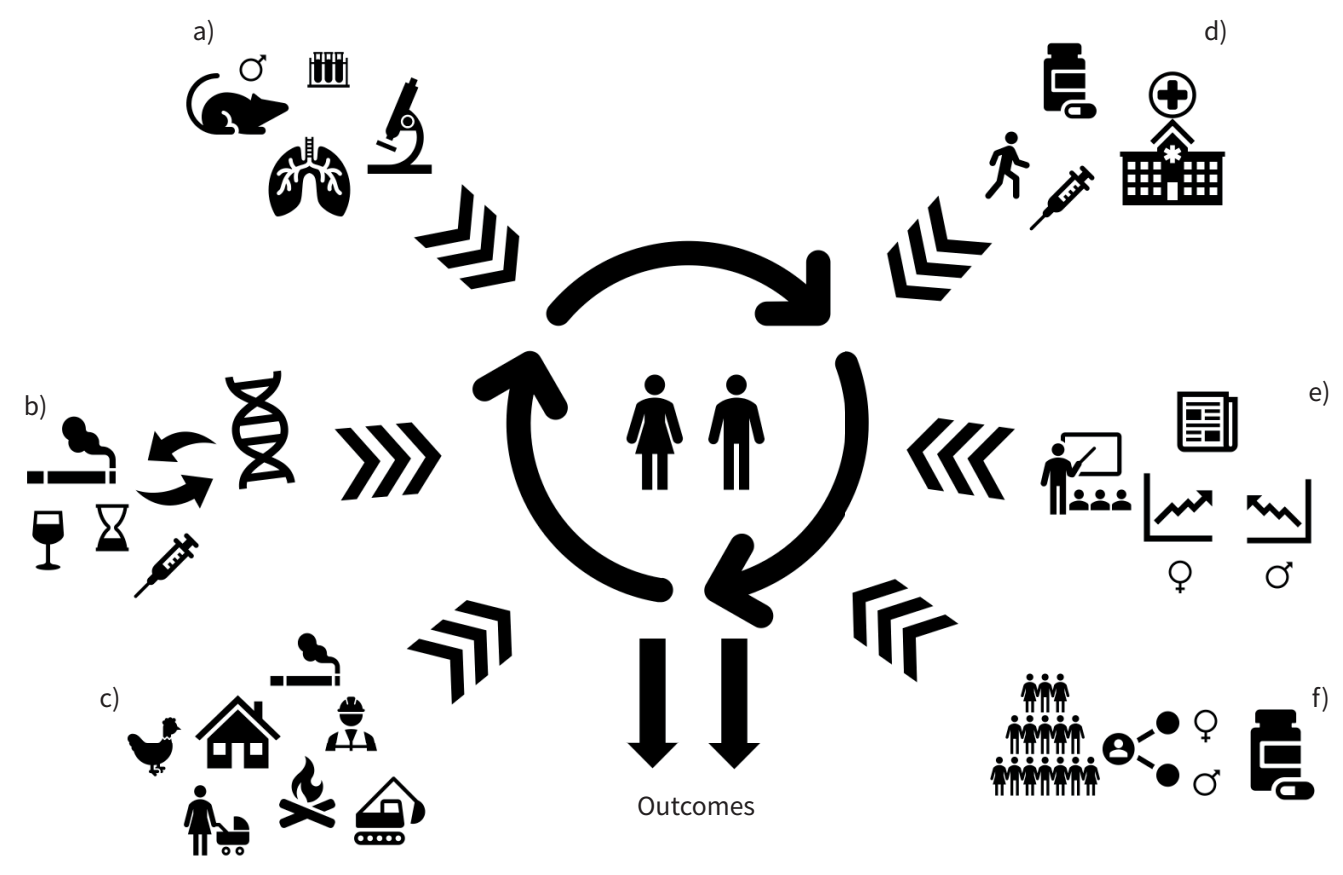

FIGURE 1 Complex interplay of sex and gender influencing health outcomes. a) Animal models of pulmonary fibrosis are exclusively performed in male mice; b) genetic and epigenetic determinants of sex and/or gender differences in interstitial lung diseases (ILDs); c) environmental and occupational exposures are highly gendered; d) healthcare utilisation and access are gendered and impact outcomes; e) sex and gender needs to be incorporated in the ILD fellowship training towards a more patient-centred medicine; f) clinical research needs to be stratified on sex/gender in order to avoid missing possible gender/sex effect modification on outcomes.

interaction with healthcare systems. These domains interact with one another in a multidimensional way, contributing to the diversity of the sex and gender spectra in disease (figure 1) [5].

In this article, we aim to address sex and gender as effect modifiers of interstitial lung diseases (ILDs), providing clinicians and researchers in the field with a current and comprehensive review of the topic. As much as possible, the words female and male will be used to refer to sex-related differences, while women and men will be used to refer to gender-related differences. Attention to the role of sex and gender differences in ILD will improve research design, assessment of outcomes and individualised patient-centred clinical practice.

\section{Epidemiology}

There are established sex differences in the incidence and prevalence of many ILDs: 50000 adults aged $\geqslant 60$ years are diagnosed annually with idiopathic pulmonary fibrosis (IPF), with $>50000$ annual deaths worldwide [6, 7]. Older age, past history of smoking and male sex (1.5- to two-fold more than females) are associated with higher incidence of IPF and shorter survival time [8]. While the aetiology of IPF remains incompletely elucidated, there is a strong association between IPF and reduction in telomere length, intimately linking IPF to ageing. Males aged $>65$ years have the highest mortality and disease prevalence [9], and IPF is commonly considered a male-predominant disease.

Several ILDs are directly caused by occupational exposures, including silicosis, asbestosis, coal workers' pneumoconiosis and berylliosis. Other forms of ILD have robust associations with certain job-related exposures, particularly hypersensitivity pneumonitis and IPF. There are more men in industrial and labour workforces with higher risk of exposures to silica, asbestos and hard metals, and thus their associated ILDs $[10,11]$. In addition, men are more likely to be exposed to vapours, gases, dusts and fumes [12], an exposure grouping with an estimated population-attributable fraction of $26 \%$ for IPF [13]. Historically, research in occupational lung disease has focused on men's health, introducing challenges and potential biases in characterising women's risks in specific workplaces, and subsequent diagnostic delays [14, 15]. Furthermore, women are more likely to have secondary or indirect exposures associated with ILD, 
including asbestos and second-hand smoke [16, 17]. Air pollution exposure is associated with risk of developing or exacerbating IPF [18, 19]. Gender may alter the effects of air pollution exposure with differential impacts on men and women, via effect mitigation by smoking status and activity patterns [20]. Indoor air pollution is a gendered exposure with differential exposures to women in domestic environments using biomass fuels for cooking and heating of homes [21, 22]. The impact of domestic pollution on risk of ILD requires further study.

Tobacco and cigarette use are established risk factors for the smoking-related subtypes of ILD, as well as in patients with IPF and the syndrome of combined pulmonary fibrosis and emphysema. Smoking is strongly associated with an increased risk of IPF, particularly with a >20 pack-year smoking history [23-25]. The radiographic and histopathologic features of desquamative interstitial pneumonia, respiratory bronchiolitis (RB)-associated ILD, pulmonary Langerhans' cell histiocytosis and smoking-related interstitial fibrosis are identified almost exclusively in former or current smokers [26-28]. Smoking is a gendered behaviour, with differential smoking rates between men and women and patterns varying according to cultural norms, targeted marketing and duration [29, 30]. Despite declining overall tobacco use, smoking remains the leading risk factor for chronic respiratory disease-related disability among men worldwide [29]. While smoking has typically been highest in men, this gender gap narrowed over recent decades in both high and lower income countries, due to targeted marketing and changing social norms [30]. Of concern, these trends may lead to higher prevalence of smoking-related lung disease, including ILD, in women. The widespread and rising use of electronic cigarettes and vaping products cause concern as such products are associated with increased tobacco use [31, 32] and acute lung injury [33], with unknown long-term pulmonary effects. Gendered marketing campaigns for smoking and vaping, and those targeted at youth pose a substantial health risk for future generations.

From what is known about asthma, COPD and lung cancer, it is highly likely that the role of tobacco and other exposures in ILD is not only mediated by sociocultural gendered behaviours, but also by sex: airway diameter, sex hormones and enzyme metabolism are different between males and females [34]. Nevertheless, the exact mechanisms behind these effects on ILD are not yet fully understood.

In regard to specific ILDs, HP deserves special mention. HP is an ILD resulting from an immune-mediated response in susceptible and sensitised individuals to a large variety of inhaled antigens found in the environment, and is more common in males in some case series. In the most recent population-based epidemiological studies in the United States, HP predominates among women (58\%) [35]. However, reports from other countries, including Denmark, report a predominance of disease among men (57\%) [36]. Interestingly, while HP occurs in a relatively gender-balanced prevalence, the aetiologies of HP may differ by gender, especially as exposure to certain aetiologic antigens may be gender-biased in occupational HP. A meta-analysis of 15 studies identified a population-attributable fraction of $19 \%$ for occupational exposures leading to HP [13], and metal-working fluid exposures account for more than half of all occupational HP cases in the United Kingdom [37]. Men appear to be more likely than women to develop HP due to farming or metal-working fluid exposures, but there is a paucity of data specifically analysing gendered risks of exposures for the development of HP [38, 39]. HP is less frequent in smokers or ex-smokers, although these data are not reported in relationship to sex and gender. Many cases are associated with an occupational exposure, while others may have no identified antigen [40]. The majority of cases of HP are related to bird exposure [40]. Antigen-indeterminate cases are reported more often in older women with lower lung function, less alveolar lymphocytosis and greater fibrosis on lung imaging [41].

The connective tissue disease (CTD)-associated ILDs also have some gender/sex nuances that deserve special mention. CTD patients who develop CTD-ILD are more frequently younger women and never-smokers [42]. The greatest sex difference, with a ratio of women to men of 7:1-10:1, is observed in systemic lupus erythematosus, and in other CTDs frequently associated with ILD: systemic sclerosis, rheumatoid arthritis and Sjögren's syndrome [5, 42]. In rheumatoid arthritis-associated ILD, interestingly, although rheumatoid arthritis predominates in women, the usual interstitial pneumonia (UIP) pattern is noted more frequently in men, whereas the non-UIP pattern is reported more in women [43, 44].

Finally, lymphangioleiomyomatosis (LAM) is the ILD with the strongest sex influence. It is basically a female disease affecting mostly white women of childbearing age [45, 46]. LAM can be considered a low-grade neoplasm and has been included in the family of "perivascular epithelioid cell tumours", a heterogeneous group of mesenchymal tumours [47]. LAM is found in a sporadic form or in conjunction with mutations in the tuberous sclerosis complex genes leading to constitutive activation of the rapamycin (mTOR) pathway [46]. Both forms can lead to progressive respiratory failure. LAM can present in organs other than the lung, causing lymphatic manifestations including lymphangioleiomyomas and 
angiomyolipomas involving the kidney [45]. The worldwide prevalence of LAM has been estimated at 3.4-7.8 women per million [47]. Oestrogens are believed to play a pathogenic role in LAM, since it is restricted almost exclusively to women, is exacerbated by exogenous oestrogen use including oral contraceptives, worsens with the onset of menses [48] and pregnancy [49-51], and is less aggressive in post-menopausal women [51]. However, hormonal-related treatment modalities lack efficacy and their use has been discouraged by expert guidelines [52]. Additionally, the rare reports of LAM in men include a male with high levels of aromatase, an enzyme that converts androgens to oestrogen, further supporting the role of oestrogens/oestrogen receptors in LAM [53].

\section{Sex as an effect modifier in ILD}

\section{Animal models}

Historically, pre-clinical studies excluded female animals due to the recognised but poorly understood influence of the reproductive cycle on experimental conduct and data [54]. Subsequent studies demonstrate that males present equal or greater variability than females owing to fluctuations in testosterone levels and other factors including caging conditions [55]. There are no differences in measures of gene expression in microarray data between males and females in mice and human studies [56]. However, sex differences can help to explain variability in data and to reduce the overall number of experiments required to determine significant outcomes. For instance, in ILD, the majority of studies investigating pulmonary fibrosis to date utilise young male bleomycin (Bleo)-treated mice, aged 8-12 weeks [57-59]. Other animal models of fibrotic lung disease do exist. West Highland Terriers (Westies), cats, donkeys, ferrets and horses do develop ILD [60, 61]. However, the animal model mostly used is the Bleo-treated mouse. Spontaneous resolution of Bleo-induced pulmonary fibrosis is observed in young mice, a phenomenon not observed in aged mice [62-64]. Mirroring the outcomes of elderly men with IPF, REDENTE et al. [65] noted that aged Bleo-treated male mice developed more severe lung disease with higher mortality compared to aged female mice. Thus, the use of aged male mice has been thought to provide a clinically relevant animal model of IPF.

Oestrogens have been postulated to play a significant role in the sex differences noted in the pulmonary fibrosis animal model. Studies evaluating the immune reactivity and inflammatory response in males and females have suggested an influence of oestrogen on disease severity [66]. In one model of pulmonary fibrosis where age-matched C57BL/6 female and male mice were treated with Bleo, a greater reduction in static lung compliance (a surrogate of fibrosis/inflammation) was observed in the lungs of male mice compared to females (50\% versus 10\%) [67]. Furthermore, when male mice were castrated, this reduction in static compliance was attenuated, while when female mice were treated with dihydrotestosterone, a similar effect on compliance as observed in male mice was seen [67]. While oestrogens may play a role in attenuating disease, other findings suggest the involvement of androgens. Irrespective of the exact causes for the sex differences observed in pulmonary fibrosis animal models, these data inform that the sex of the animal influences experimental results.

Although the inclusion of female and male animals appears to require a doubling in the number of experimental participants, more efficient experimental/adaptive designs can incorporate both sexes without increasing variance excessively [68]. A successful strategy can use factorial designs, in which two experimental factors with multiple levels are tested and data are collected across all possible combinations of factors and levels. This enables the effect of each factor to be tested in addition to the interaction between the factor levels. Sample sizes may need to be increased by $14-33 \%$ to account for the estimation of the extra parameter [69].

\section{Genetics}

There is a 10-fold increase in prevalence of IPF in some families of patients with a diagnosis of IPF, supporting a genetic predisposition to ILD [70, 71]. Genetic studies of familial forms of ILD led to the discovery of mutations in genes implicated in telomere-related gene (TRG) homeostasis or surfactant homeostasis associated with complex syndromes [72]. Loss-of-function TRG mutations are associated with shortening of telomere length [73, 74]. The development of ILD and whether telomere length is a biomarker of increased severity of disease in TRG mutation carriers remains unknown, although the telomere length by itself, whatever the presence of a TRG mutation, might be implicated in the ILD pathophysiology by inducing a senescence programme. Indeed, telomere length could be a biomarker of increased severity of the disease in addition to the presence of a germline mutation [73, 75].

Among all the known TRG mutations associated with ILD, only DKC1 is located on the sex chromosomes, more specifically in the X chromosome, leading to X-linked dyskeratosis congenita, where only males are affected. All the other known TRG mutations are located on autosomes [76]. 
In patients with ILD and TRG mutations, men are usually younger than women (54 versus 63 years, respectively) at diagnosis, and the prevalence of ILD increases with age [77]. Telomeres are known to progressively shorten with age and environmental and occupational exposures vary by gender. These data imply that gender, sex, sex hormones and age may contribute to the development of ILD [75, 77, 78].

In a reported cohort of familial pulmonary fibrosis, telomere length was significantly shorter in diseased men, indicating that women might be protected from telomere shortening [79]. Blood cell counts of TRG mutation carriers can improve with androgen therapy [80]. In vitro androgens increase telomerase activity and TERT concentration mRNA levels in control cells, and restore telomerase activity in cells from TRG-mutation carriers [81]. Oestradiol had a similar effect in an oestrogen receptor (ER)- $\alpha$ dependent manner. Further in vitro studies suggest that regulation of telomerase expression and activity is mediated by aromatisation of androgens and ER- $\alpha$ [81].

Danazol, a synthetic sex hormone with androgenic properties, showed promise as a treatment for pulmonary fibrosis associated with TRG mutations [80]. Treatment with danazol promoted telomere elongation and favourable haematological response in $79 \%$ of the cases (19 out of 24); however, more women than men (15 out of 27) were included in this trial and the median age at inclusion was 41 years [80]. A clinical trial with danazol is currently ongoing in France for patients with ILD and TRG mutations [82] (clinicaltrials.gov NCT03710356).

There is growing evidence that genetic variation on autosomes (outside the sex chromosomes) occurs in a sex-dependent matter [83]. Furthermore, heritability unexplained by genetic variation may be attributable to environmental factors that drive epigenetic control of gene expression such as endogenous biological factors (e.g. hormones and enzymes) and environmental factors with behavioural gender differences (e.g. smoking, environmental toxins) [84].

Future genomic studies should include sex- and gender-specific questions. How does sex influence the risk of ILD and disease prognosis for ILD-associated mutation carriers? Does hormonal treatment and menopause modify telomere length and the prevalence of ILD in TRG mutation carriers? Are there sex-specific pharmacogenetic differences modifying the effect of antifibrotic drugs? [85].

The influence of genomics in disease phenotype may vary; for some diseases the contribution of genetic factors is very high (determinant), while for others genetic factors are less important and largely influenced by other factors (environmental, behavioural) in order for disease to manifest [86]. As outlined earlier, sex, expressed by the action of sex hormones and sexually regulated genetic and epigenetic phenomena, explains part but not all of the variability seen between females and males with ILD. The other part is due to behaviours that are different across gender, leading to different exposures, healthcare-seeking patterns and healthcare provision.

\section{Gender as an effect modifier in ILD}

Beyond the effect of biological sex in ILD, gender may be an important effect modifier in patients with ILD, impacting the risk of developing disease, the likelihood of obtaining a diagnosis or receiving treatment and specific clinical outcomes including overall quality of care and survival. It is important to note that many of the studies cited herein use the terms sex and gender synonymously, and it is challenging to elucidate whether the variables intend to describe biological sex or gender. Our review underscores the importance of inclusion and specification of sex and gender in all studies, avoiding assumptions that male and female data are interchangeable and the influence of gender is predictable.

\section{Diagnosis}

Given the consistent and well-described predominance of men in IPF, gender appears to also influence diagnostic impression of ILD by physicians, whereby men who have high-resolution computed tomography patterns other than definite UIP are more likely to be given a diagnosis of IPF [87]. Despite increased awareness of IPF among men, delays are still seen and were associated with trials of inhaled therapies, better lung physiology and better scores in quality-of-life questionnaires [88]. The combination of male sex and age $>60$ years yielded a specificity $>91 \%$ for underlying histopathologic UIP in another study of patients with non-definitive computed tomography patterns [89]. The Australian IPF Registry has reported a definite UIP pattern more commonly in men, while there were relatively more women meeting criteria for possible UIP or inconsistent with UIP patterns [90]. 


\section{Prognosis}

Men tend to have a worse prognosis for IPF and other non-IPF ILDs, with lower transplant-free survival [91]. This strong signal has been well validated using the GAP (gender, age, physiology) score, where gender (being a man) confers a worse prognosis in IPF and in non-IPF ILD [92, 93]. US claims data have also shown that overall mortality rates of IPF for men were 1.63 times that of women [94]. Using German administrative claims data of $>36000$ individuals, WäLSCHER et al. [95] found gender-based differences in number of hospitalisations in patients with ILD. In their study, women had significantly lower risks of both ILD-related and non-ILD-related hospitalisations. Men are at increased risk of faster disease progression and increased mortality in different CTD-ILDs as well, including systemic sclerosis [96], rheumatoid arthritis [97, 98] and autoimmune inflammatory myopathy [99]. In a single-centre study of 243 patients with CTD-ILD, men experienced increased mortality across diseases [100]. It is unknown whether these differences in prognosis are mainly influenced by biological sex factors, behavioural gender or both.

\section{Medication usage}

Recent data indicate that gender impacts the probability of being on disease-targeted therapy for ILD, although these studies did not report gender specifically, considering sex instead as the dependent variable. However, given the sociocultural factors of health behaviours and healthcare provision, we consider gender here to be a more influential metric than biological sex. In a large multicentre Canadian registry of patients with fibrotic ILD, male sex and lower lung function were associated with shorter time to treatment initiation in the overall cohort (hazard ratio (HR) 1.24, 95\% CI 1.05-1.48) and in the subgroup of patients with IPF (HR 1.85, 95\% CI 1.28-2.66) [101]. In a US administrative claims-based cohort of nearly 11000 patients with IPF, only one-quarter of patients started an antifibrotic medication during the study period [102]. Of those treated, men were significantly more likely than women to be on an antifibrotic (30\% versus $21.9 \%, \mathrm{p}<0.0001$ ). The out-of-pocket costs for treatment were high at USD 400 per month, but it is unclear whether financial barriers may have contributed to the differential use of antifibrotics in this population, and specifically the differences by gender. In a longitudinal study of Korean patients with IPF, men were more likely than women to be treated with antifibrotics (80\% versus $73 \%$ ), a difference less likely to be attributable to financial differences given their universal health coverage [103]. Given the prevailing data that male sex predicts worse outcomes in patients with IPF, treatment decisions may be influenced by the perceived risk of progression [92]. This may explain why men are more often treated with antifibrotics compared to women, but may not be justifiable given the known natural history of untreated IPF and the recommendation that antifibrotics should be considered for all patients [104, 105]. The reasons underlying gendered differences in ILD treatments warrant further study to identify and address systemic barriers in equitable access to treatments. Overall, women have been found less likely to receive guideline-based drug therapy and to adhere to medications for chronic conditions [106], an issue of critical importance for lung diseases like ILD.

These gender-based treatment differences extend to nonpharmacological therapies such as exercise rehabilitation, where men make up a greater proportion of the study population in pulmonary rehabilitation studies [107, 108]. Although lower adherence is observed among women, they usually seek more healthcare in response to physical and mental symptoms, and that argues against intentional nonadherence or disease denial as possible explanations for inferior adherence as compared to men [109]. To the contrary it seems that men and women are managed differently in the healthcare system: women present more frequently with atypical symptoms, making it more difficult to reach a diagnosis [110]. Additionally, women are considered at lower risk for certain diseases (for example, for IPF or acute myocardial infarction), which makes physicians less aware, decreasing adherence to medical treatment. Males tend to be included in clinical trials of IPF more frequently than females, as manifested in trials with antifibrotic therapies [111-114]. A similar disparity was found among lung transplant recipients, with men receiving more transplants than women with IPF, despite higher risk of death in males post-transplant $[115,116]$.

\section{Sex and gender in research}

Integrating sex and gender analysis into the design of research, where relevant, can lead to discovery and improved research methodology [1]. The goal of sex and gender analysis is to promote rigorous, reproducible and responsible science. It is crucial that data be stratified on the level of sex and/or gender. This will improve accuracy and avoid misinterpretations and sex-level generalisabilities where not appropriate. The common practice of pooling results for females and males or women and men can mask important sex differences.

Much work remains to be done to systematically integrate sex and gender analysis into relevant domains of science, from strategic considerations for establishing research priorities to guidelines for establishing best practices in formulating research questions, designing methodologies and interpreting data. To achieve 
TABLE 1 Suggested topics for inclusion in interstitial lung disease (ILD) fellowship training

Overall review of sex and gender-based medicine

Sex influence in pulmonary fibrosis animal models

Sex and gender influences in genetics and epigenetics in ILD

Gendered behaviours influencing exposures in ILD

Gendered behaviours influencing healthcare system utilisation and healthcare provision

Sex and gender in the research agenda (designs accounting for this additional stratification)

Gender bias in career progression of women physicians, and importance of equity, diversity and inclusion

Appropriate references for each topic are provided throughout the text.

meaningful progress in the next decade, researchers, funding agencies, peer-reviewed journals and universities should coordinate efforts to develop and standardise methods of sex and gender analysis. Accounting for sex and gender is essential to individualised medicine aiming to 1) delineate and investigate sex- and gender-based differences in health, disease and response to treatment and 2) apply that knowledge to clinical care to improve the health of both women and men [5, 117].

Sex and gender in ILD education and careers

Sex and gender-based medicine (SGBM) should be considered essential to better preparing future ILD physicians. However, the integration of SGBM into the ILD fellowship training is poorly defined; schools often do not know the current status of SGBM content in their curricula, and this is even worse for subspecialties training. An important step to push forward SGBM in ILD training is first to recognise sexand gender-based differences in health, disease and response to treatment, and second to officially incorporate SGBM into the ILD training curriculum. Suggested items to be included in ILD fellowship curricula are listed in table 1.

While gender inequality in medicine may be unintentional, research suggests that it is the effect of both implicit and explicit biases [118-120]. Sociocultural factors also contribute, especially for those with parental, caregiver and home responsibilities, as do considerations of race and ethnicity. Gender inequity is ubiquitous across private and public sectors, suggesting that its causes relate to structural and systemic barriers, rather than individual preference. For instance, a recent publication showed that no women led as first or senior author any of the major industry-sponsored trials or society-sponsored clinical practice guidelines within the past 10 years in the ILD field [121]. Industry-sponsored trials and society-sponsored guidelines are activities where participation occurs by invitation, which reflects power and network.

The paucity of women in leadership roles reflects systemic biases in academia and medicine that promote and perpetuate "leaky pipeline" and "glass ceiling" effects [122]. While over half of graduating medical students have been women in the past two decades, they still represent a disproportionate minority of medical school deans [123], department chairs, division chiefs and full professors [124]. A robust and growing body of evidence demonstrates gender inequity in conference presentations and authorship of peer-reviewed publications across diverse fields [121, 125, 126]. Professional medical societies, editorial boards and pharmaceutical companies must continue to evaluate and revise their processes to ensure continued growth towards diversity in representation, including inclusion across domains of gender, LGBTQ+, race and ethnicity [127]. Deserving specific mention are transgender and gender non-binary individuals (TGGNB), since these are among the most stigmatised individuals in the US, and probably in most other countries $[128,129]$. The term TGGNB is used to refer to individuals who express their gender in ways that differ from the established cultural norms where gender is defined from sex assigned at birth. The social marginalisation of TGGNB individuals has a significant impact on their health and quality of life [130]. TGGNB people experience significantly high rates of poor physical and mental health [129]. When TGGNB individuals do have access to healthcare, they frequently experience stigma and discrimination from providers [129]. Healthcare providers need to be trained to offer proper healthcare to these individuals while institutional and system-level changes are implemented to support providers in their abilities to care for TGGNB patients. Collecting data on TGGNB status in ILD research will further help inform the unique issues in this patient population.

Conclusions and future perspectives

Our understanding of the interplay between sex and gender is imperfect and evolving; greater consideration of sex and gender is needed in basic and clinical ILD research. Many gaps in knowledge still remain. The goals are to improve care for all ILD patients in light of identified sex and gender. This review 
demonstrates the importance of sex and gender aspects of disease and the attention needed to biological and social determinants of health in training curricula for clinicians and researchers. We encourage ILD physicians to consider sex and gender broadly across their practice to optimise care for all patients, disseminate up-to-date knowledge, advance research where gaps exist and educate the next generation of ILD clinicians to acknowledge and incorporate sex and gender in the management of health and disease.

Provenance: Commissioned article, peer reviewed.

Previous articles in this series: No. 1: Cheron C, McBride SA, Antigny F, et al. Sex and gender in pulmonary arterial hypertension. Eur Respir Rev 30: 2021; 200330. No. 2: LoMauro A, Aliverti A. Sex and gender in respiratory physiology. Eur Respir Rev 30: 2021; 210038. No. 3: Chowdhury NU, Guntur VP, Newcomb DC, et al. Sex and gender in asthma. Eur Respir Rev 30: 2021; 210067.

Conflict of interest: None declared.

\section{References}

1 Tannenbaum C, Ellis RP, Eyssel F, et al. Sex and gender analysis improves science and engineering. Nature 2019; 575: 137-146.

2 Seaborn T, Simard M, Provost PR, et al. Sex hormone metabolism in lung development and maturation. Trends Endocrinol Metab 2010; 21: 729-738.

3 Mauvais-Jarvis F, Bairey Merz N, Barnes PJ, et al. Sex and gender: modifiers of health, disease, and medicine. Lancet 2020; 396: 565-582.

4 McGregor AJ, Greenberg M, Safdar B, et al. Focusing a gender lens on emergency medicine research: 2012 update. Acad Emerg Med 2013; 20: 313-320.

5 Bartz D, Chitnis T, Kaiser UB, et al. Clinical advances in sex- and gender-informed medicine to improve the health of all: a review. JAMA Intern Med 2020; 180: 574-583.

6 Martinez FJ, Collard HR, Pardo A, et al. Idiopathic pulmonary fibrosis. Nat Rev Dis Primers 2017; 3: 17074.

7 Raghu G, Weycker D, Edelsberg J, et al. Incidence and prevalence of idiopathic pulmonary fibrosis. Am J Respir Crit Care Med 2006; 174: 810-816.

8 Salisbury ML, Xia M, Zhou Y, et al. Idiopathic pulmonary fibrosis: gender-age-physiology index stage for predicting future lung function decline. Chest 2016; 149: 491-498.

9 Sauleda J, Núñez B, Sala E, et al. Idiopathic pulmonary fibrosis: epidemiology, natural history, phenotypes. Med Sci 2018; 6: 110.

10 Poinen-Rughooputh S, Rughooputh MS, Guo Y, et al. Sex-related differences in the risk of silicosis among Chinese pottery workers: a cohort study. J Occup Environ Med 2021; 63: 74-79.

11 Shi P, Xing X, Xi S, et al. Trends in global, regional and national incidence of pneumoconiosis caused by different aetiologies: an analysis from the Global Burden of Disease Study 2017. Occup Environ Med 2020; 77: 407-414.

12 Blanc PD, Eisner MD, Balmes JR, et al. Exposure to vapors, gas, dust, or fumes: assessment by a single survey item compared to a detailed exposure battery and a job exposure matrix. Am J Ind Med 2005; 48: 110-117.

13 Blanc PD, Annesi-Maesano I, Balmes JR, et al. The occupational burden of nonmalignant respiratory diseases. An Official American Thoracic Society and European Respiratory Society Statement. Am J Respir Crit Care Med 2019; 199: 1312-1334.

14 Camp PG, Dimich-Ward H, Kennedy SM. Women and occupational lung disease: sex differences and gender influences on research and disease outcomes. Clin Chest Med 2004; 25: 269-279.

15 Kerget B, Araz O, Yilmazel Ucar E, et al. Female workers' silicosis diagnosis delayed due to gender bias. Occup Med 2019; 69: 219-222.

16 Panou V, Vyberg M, Meristoudis C, et al. Non-occupational exposure to asbestos is the main cause of malignant mesothelioma in women in North Jutland, Denmark. Scand J Work Environ Health 2019; 45: 82-89.

17 Abramson MJ, Murambadoro T, Alif SM, et al. Occupational and environmental risk factors for idiopathic pulmonary fibrosis in Australia: case-control study. Thorax 2020; 75: 864-869.

18 Conti S, Harari S, Caminati A, et al. The association between air pollution and the incidence of idiopathic pulmonary fibrosis in Northern Italy. Eur Respir J 2018; 51 :1700397.

19 Johannson KA, Vittinghoff E, Lee K, et al. Acute exacerbation of idiopathic pulmonary fibrosis associated with air pollution exposure. Eur Respir J 2014; 43: 1124-1131.

20 Clougherty JE. A growing role for gender analysis in air pollution epidemiology. Environ Health Perspect 2010; 118: 167-176. 
Po JY, FitzGerald JM, Carlsten C. Respiratory disease associated with solid biomass fuel exposure in rural women and children: systematic review and meta-analysis. Thorax 2011; 66: 232-239.

Siddharthan T, Grigsby MR, Goodman D, et al. Association between household air pollution exposure and chronic obstructive pulmonary disease outcomes in 13 low- and middle-income country settings. Am $J$ Respir Crit Care Med 2018; 197: 611-620.

Iwai K, Mori T, Yamada N, et al. Idiopathic pulmonary fibrosis. Epidemiologic approaches to occupational exposure. Am J Respir Crit Care Med 1994; 150: 670-675.

24 Baumgartner KB, Samet JM, Stidley CA, et al. Cigarette smoking: a risk factor for idiopathic pulmonary fibrosis. Am J Respir Crit Care Med 1997; 155: 242-248.

25 Taskar VS, Coultas DB. Is idiopathic pulmonary fibrosis an environmental disease? Proc Am Thorac Soc 2006; 3: $293-298$

26 Travis WD, Costabel U, Hansell DM, et al. An official American Thoracic Society/European Respiratory Society statement: update of the international multidisciplinary classification of the idiopathic interstitial pneumonias. Am J Respir Crit Care Med 2013; 188: 733-748.

Hellemons ME, Moor CC, von der Thüsen J, et al. Desquamative interstitial pneumonia: a systematic review of its features and outcomes. Eur Respir Rev 2020; 29: 190181.

28 Flaherty KR, Fell C, Aubry MC, et al. Smoking-related idiopathic interstitial pneumonia. Eur Respir J 2014; 44: 594-602.

GBD Chronic Respiratory Disease Collaborators. Prevalence and attributable health burden of chronic respiratory diseases, 1990-2017: a systematic analysis for the Global Burden of Disease Study 2017. Lancet Respir Med 2020; 8: 585-596.

Samet JM, Yoon S-Y, World Health Organization. Gender, Women, and the Tobacco Epidemic. 2010. Available from: https://apps.who.int/iris/handle/10665/44342

31 Besaratinia A, Tommasi S. Vaping: a growing global health concern. EClinicalMedicine 2019; $17: 100208$. (the Risks of E-cigarettes for Kids, Teens, and Young Adults. www.cdc.gov/tobacco/basic_information/e-cigarettes/Quick-Facts-on-the-Risks-of-E-cigarettesfor-Kids-Teens-and-Young-Adults.html Date last updated: 16 December 2020.

Koslow M, Petrache I. A finale on EVALI?: the abated but not forgotten outbreak of acute respiratory illness in individuals who vape. JAMA Netw Open 2020; 3: e2019366.

34 Rebuli ME. Respiratory sex differences in response to smoke exposure. In: Silveyra P, Tingo XT, eds. Sex-Based Differences in Lung Physiology. Cham, Springer, 2021; pp. 291-321.

Fernández Pérez ER, Kong AM, Raimundo K, et al. Epidemiology of hypersensitivity pneumonitis among an insured population in the United States: a claims-based cohort analysis. Ann Am Thorac Soc 2018; 15: 460-469.

Rittig AH, Hilberg $\mathrm{O}$, Ibsen $\mathrm{R}$, et al. Incidence, comorbidity and survival rate of hypersensitivity pneumonitis: a national population-based study. ERJ Open Res 2019; 5: 00259-2018.

Barber CM, Wiggans RE, Carder M, et al. Epidemiology of occupational hypersensitivity pneumonitis; reports from the SWORD scheme in the UK from 1996 to 2015. Occup Environ Med 2017; 74: 528-530.

Reynolds C, Feary J, Cullinan P. Occupational contributions to interstitial lung disease. Clin Chest Med 2020; 41: 697-707.

39 Tillie-Leblond I, Grenouillet F, Reboux G, et al. Hypersensitivity pneumonitis and metalworking fluids contaminated by mycobacteria. Eur Respir J 2011; 37: 640-647.

40 Costabel U, Miyazaki Y, Pardo A, et al. Hypersensitivity pneumonitis. Nat Rev Dis Primers 2020; 6: 65.

41 Walters GI, Mokhlis JM, Moore VC, et al. Characteristics of hypersensitivity pneumonitis diagnosed by interstitial and occupational lung disease multi-disciplinary team consensus. Respir Med 2019; 155: 19-25.

Ortona E, Pierdominici M, Maselli A, et al. Sex-based differences in autoimmune diseases. Ann Ist Super Sanita 2016; 52: 205-212.

43 Sparks JA, He X, Huang J, et al. Rheumatoid arthritis disease activity predicting incident clinically apparent rheumatoid arthritis-associated interstitial lung disease: a prospective cohort study. Arthritis Rheumatol 2019; 71: 1472-1482.

44 Kass DJ, Nouraie M, Glassberg MK, et al. Comparative profiling of serum protein biomarkers in rheumatoid arthritis-associated interstitial lung disease and idiopathic pulmonary fibrosis. Arthritis Rheumatol 2020; 72: 409-419.

45 Sullivan EJ. Lymphangioleiomyomatosis: a review. Chest 1998; 114: 1689-1703.

46 Torre O, Elia D, Caminati A, et al. New insights in lymphangioleiomyomatosis and pulmonary Langerhans cell histiocytosis. Eur Respir Rev 2017; 26: 170042.

47 Harari S, Torre O, Moss J. Lymphangioleiomyomatosis: what do we know and what are we looking for? Eur Respir Rev 2011; 20: 34-44.

48 Svendsen TL, Viskum K, Hansborg N, et al. Pulmonary lymphangioleiomyomatosis: a case of progesterone receptor positive lymphangioleiomyomatosis treated with medroxyprogesterone, oophorectomy and tamoxifen. Br J Dis Chest 1984; 78: 264-271. 
Yockey CC, Riepe RE, Ryan K. Pulmonary lymphangioleiomyomatosis complicated by pregnancy. Kans Med 1986; 87: 277-278.

Eliasson AH, Phillips YY, Tenholder MF. Treatment of lymphangioleiomyomatosis. A meta-analysis. Chest 1989; 96: 1352-1355.

Johnson SR, Tattersfield AE. Clinical experience of lymphangioleiomyomatosis in the UK. Thorax 2000; 55: 1052-1057.

Gupta N, Finlay GA, Kotloff RM, et al. Lymphangioleiomyomatosis diagnosis and management: high-resolution chest computed tomography, transbronchial lung biopsy, and pleural disease management. An Official American Thoracic Society/Japanese Respiratory Society Clinical Practice Guideline. Am J Respir Crit Care Med 2017; 196: 1337-1348.

Aubry MC, Myers JL, Ryu JH, et al. Pulmonary lymphangioleiomyomatosis in a man. Am J Respir Crit Care Med 2000; 162: 749-752.

Becker JB, Prendergast BJ, Liang JW. Female rats are not more variable than male rats: a meta-analysis of neuroscience studies. Biol Sex Differ 2016; 7: 34.

Prendergast BJ, Onishi KG, Zucker I. Female mice liberated for inclusion in neuroscience and biomedical research. Neurosci Biobehav Rev 2014; 40: 1-5.

Itoh Y, Arnold AP. Are females more variable than males in gene expression? Meta-analysis of microarray datasets. Biol Sex Differ 2015; 6: 18.

Izbicki G, Segel MJ, Christensen TG, et al. Time course of bleomycin-induced lung fibrosis. Int J Exp Pathol 2002; 83: 111-119.

Peng R, Sridhar S, Tyagi G, et al. Bleomycin induces molecular changes directly relevant to idiopathic pulmonary fibrosis: a model for "active" disease. PLoS One 2013; 8: e59348.

Aguilar S, Scotton CJ, McNulty K, et al. Bone marrow stem cells expressing keratinocyte growth factor via an inducible lentivirus protects against bleomycin-induced pulmonary fibrosis. PLoS One 2009; 4: e8013.

Roman J, Brown KK, Olson A, et al. An official American Thoracic Society workshop report: comparative pathobiology of fibrosing lung disorders in humans and domestic animals. Ann Am Thorac Soc 2013; 10: S224-S229.

Williams KJ, Robinson NE, Lim A, et al. Experimental induction of pulmonary fibrosis in horses with the gammaherpesvirus equine herpesvirus 5. PLoS One 2013; 8: e77754.

Degryse AL, Tanjore H, Xu XC, et al. Repetitive intratracheal bleomycin models several features of idiopathic pulmonary fibrosis. Am J Physiol Lung Cell Mol Physiol 2010; 299: L442-L452.

Redente EF, Keith RC, Janssen W, et al. Tumor necrosis factor- $\alpha$ accelerates the resolution of established pulmonary fibrosis in mice by targeting profibrotic lung macrophages. Am J Respir Cell Mol Biol 2014; 50: 825-837.

Chung MP, Monick MM, Hamzeh NY, et al. Role of repeated lung injury and genetic background in bleomycin-induced fibrosis. Am J Respir Cell Mol Biol 2003; 29: 375-380.

Redente EF, Jacobsen KM, Solomon JJ, et al. Age and sex dimorphisms contribute to the severity of bleomycin-induced lung injury and fibrosis. Am J Physiol Lung Cell Mol Physiol 2011; 301: L510-L518.

Gharaee-Kermani M, Hatano K, Nozaki Y, et al. Gender-based differences in bleomycin-induced pulmonary fibrosis. Am J Pathol 2005; 166: 1593-1606.

Voltz JW, Card JW, Carey MA, et al. Male sex hormones exacerbate lung function impairment after bleomycin-induced pulmonary fibrosis. Am J Respir Cell Mol Biol 2008; 39: 45-52.

Beery AK. Inclusion of females does not increase variability in rodent research studies. Curr Opin Behav Sci 2018; 23: 143-149.

Buch T, Moos K, Ferreira FM, et al. Benefits of a factorial design focusing on inclusion of female and male animals in one experiment. J Mol Med 2019; 97: 871-877.

Hodgson U, Laitinen T, Tukiainen P. Nationwide prevalence of sporadic and familial idiopathic pulmonary fibrosis: evidence of founder effect among multiplex families in Finland. Thorax 2002; 57: 338-342.

Kropski JA, Pritchett JM, Zoz DF, et al. Extensive phenotyping of individuals at risk for familial interstitial pneumonia reveals clues to the pathogenesis of interstitial lung disease. Am J Respir Crit Care Med 2015; 191: 417-426.

72 Borie R, Kannengiesser C, de Fontbrune FS, et al. Management of suspected monogenic lung fibrosis in a specialised centre. Eur Respir Rev 2017; 26: 160122.

Collopy LC, Walne AJ, Cardoso S, et al. Triallelic and epigenetic-like inheritance in human disorders of telomerase. Blood 2015; 126: 176-184.

Alder JK, Hanumanthu VS, Strong MA, et al. Diagnostic utility of telomere length testing in a hospital-based setting. Proc Natl Acad Sci USA 2018; 115: E2358-E2365.

Newton CA, Batra K, Torrealba J, et al. Telomere-related lung fibrosis is diagnostically heterogeneous but uniformly progressive. Eur Respir J 2016; 48: 1710-1720.

Knight SW, Heiss NS, Vulliamy TJ, et al. X-linked dyskeratosis congenita is predominantly caused by missense mutations in the DKC1 gene. Am J Hum Genet 1999; 65: 50-58. 
De Leon AD, Cronkhite JT, Katzenstein A-LA, et al. Telomere lengths, pulmonary fibrosis and telomerase (TERT) mutations. PLoS One 2010; 5: e10680.

Borie R, Tabèze L, Thabut G, et al. Prevalence and characteristics of TERT and TERC mutations in suspected genetic pulmonary fibrosis. Eur Respir J 2016; 48: 1721-1731.

Cronkhite JT, Xing C, Raghu G, et al. Telomere shortening in familial and sporadic pulmonary fibrosis. Am J Respir Crit Care Med 2008; 178: 729-737.

Townsley DM, Dumitriu B, Liu D, et al. Danazol treatment for telomere diseases. N Engl J Med 2016; 374: 1922-1931.

Calado RT, Yewdell WT, Wilkerson KL, et al. Sex hormones, acting on the TERT gene, increase telomerase activity in human primary hematopoietic cells. Blood 2009; 114: 2236-2243.

Frontbrune FS, Resche-Rigon M. Assistance Publique - Hôpitaux de Paris. Evaluating Efficacy and Safety of Danazol in Severe Hematologic or Pulmonary Disease Related to Telomeropathy (ANDROTELO). NCT03710356. Available from: https://clinicaltrials.gov/ct2/show/NCT03710356/ Date last accessed: June 2021.

Dimas AS, Nica AC, Montgomery SB, et al. Sex-biased genetic effects on gene regulation in humans. Genome Res 2012; 22: 2368-2375.

Khramtsova EA, Davis LK, Stranger BE. The role of sex in the genomics of human complex traits. Nat Rev Genet 2019; 20: 173-190.

Oldham JM, Noth I, Martinez FJ. Pharmacogenetics and interstitial lung disease. Curr Opin Pulm Med 2016; 22: 456-465.

Craig J. Complex diseases: research and applications. Nat Educ 2008; 1: 184.

Assayag D, Morisset J, Johannson KA, et al. Patient gender bias on the diagnosis of idiopathic pulmonary fibrosis. Thorax 2020; 75: 407-412.

Hoyer N, Prior TS, Bendstrup E, et al. Risk factors for diagnostic delay in idiopathic pulmonary fibrosis. Respir Res 2019; 20: 103.

Brownell R, Moua T, Henry TS, et al. The use of pretest probability increases the value of high-resolution CT in diagnosing usual interstitial pneumonia. Thorax 2017; 72: 424-429.

Jo HE, Glaspole I, Goh N, et al. Implications of the diagnostic criteria of idiopathic pulmonary fibrosis in clinical practice: analysis from the Australian Idiopathic Pulmonary Fibrosis Registry. Respirology 2019; 24: 361-368.

Zaman T, Moua T, Vittinghoff E, et al. Differences in clinical characteristics and outcomes between men and women with idiopathic pulmonary fibrosis: a multicenter retrospective cohort study. Chest 2020; 158: 245-251.

Ley B, Ryerson CJ, Vittinghoff E, et al. A multidimensional index and staging system for idiopathic pulmonary fibrosis. Ann Intern Med 2012; 156: 684-691.

Ryerson CJ, Vittinghoff E, Ley $\mathrm{B}$, et al. Predicting survival across chronic interstitial lung disease: the ILD-GAP model. Chest 2014; 145: 723-728.

Olson AL, Swigris JJ, Sprunger DB, et al. Rheumatoid arthritis-interstitial lung disease-associated mortality. Am J Respir Crit Care Med 2011; 183: 372-378.

Wälscher J, Witt S, Schwarzkopf L, et al. Hospitalisation patterns of patients with interstitial lung disease in the light of comorbidities and medical treatment - a German claims data analysis. Respir Res 2020; 21: 73.

Winstone TA, Assayag D, Wilcox PG, et al. Predictors of mortality and progression in scleroderma-associated interstitial lung disease: a systematic review. Chest 2014; 146: 422-436.

Assayag D, Lubin M, Lee JS, et al. Predictors of mortality in rheumatoid arthritis-related interstitial lung disease. Respirology 2014; 19: 493-500.

Kawano-Dourado L, Doyle TJ, Bonfiglioli K, et al. Baseline characteristics and progression of a spectrum of interstitial lung abnormalities and disease in rheumatoid arthritis. Chest 2020; 158: 1546-1554.

Cobo-Ibáñez T, López-Longo F-J, Joven B, et al. Long-term pulmonary outcomes and mortality in idiopathic inflammatory myopathies associated with interstitial lung disease. Clin Rheumatol 2019; 38: 803-815.

Chartrand S, Lee JS, Swigris JJ, et al. Clinical characteristics and natural history of autoimmune forms of interstitial lung disease: a single-center experience. Lung 2019; 197: 709-713.

Assayag D, Garlick K, Johannson KA, et al. Treatment initiation in patients with interstitial lung disease in Canada. Ann Am Thorac Soc 2021; 18: 1661-1668.

Dempsey TM, Payne S, Sangaralingham L, et al. Adoption of the antifibrotic medications pirfenidone and nintedanib for patients with idiopathic pulmonary fibrosis. Ann Am Thorac Soc 2021; 18: 1121-1128.

Moon SW, Kim SY, Chung MP, et al. Longitudinal changes in clinical features, management, and outcomes of idiopathic pulmonary fibrosis. A nationwide cohort study. Ann Am Thorac Soc 2021; 18: 780-787.

Raghu G, Rochwerg B, Zhang Y, et al. An official ATS/ERS/JRS/ALAT clinical practice guideline: treatment of idiopathic pulmonary fibrosis. An update of the 2011 clinical practice guideline. Am J Respir Crit Care Med 2015; 192: e3-e19.

05 Ley B, Collard HR, King TE Jr. Clinical course and prediction of survival in idiopathic pulmonary fibrosis. Am J Respir Crit Care Med 2011; 183: 431-440. 
106 Manteuffel M, Williams S, Chen W, et al. Influence of patient sex and gender on medication use, adherence, and prescribing alignment with guidelines. $J$ Womens Health 2014; 23: 112-119.

107 Nishiyama $\mathrm{O}$, Kondoh $\mathrm{Y}$, Kimura $\mathrm{T}$, et al. Effects of pulmonary rehabilitation in patients with idiopathic pulmonary fibrosis. Respirology 2008; 13: 394-399.

108 da Fontoura FF, Berton DC, Watte G, et al. Pulmonary rehabilitation in patients with advanced idiopathic pulmonary fibrosis referred for lung transplantation. J Cardiopulm Rehabil Prev 2018; 38: 131-134.

109 Thompson AE, Anisimowicz Y, Miedema B, et al. The influence of gender and other patient characteristics on health care-seeking behaviour: a QUALICOPC study. BMC Fam Pract 2016; 17: 38.

110 Eindhoven DC, Hilt AD, Zwaan TC, et al. Age and gender differences in medical adherence after myocardial infarction: women do not receive optimal treatment - The Netherlands claims database. Eur J Prev Cardiol 2018; 25: 181-189.

111 Bargagli E, Piccioli C, Rosi E, et al. Pirfenidone and nintedanib in idiopathic pulmonary fibrosis: real-life experience in an Italian referral centre. Pulmonology 2019; 25: 149-153.

112 Tzouvelekis A, Karampitsakos T, Kontou M, et al. Safety and efficacy of nintedanib in idiopathic pulmonary fibrosis: a real-life observational study in Greece. Pulm Pharmacol Ther 2018; 49: 61-66.

113 Harari S, Caminati A, Poletti V, et al. A real-life multicenter national study on nintedanib in severe idiopathic pulmonary fibrosis. Respiration 2018; 95: 433-440.

114 Salih GN, Shaker SB, Madsen HD, et al. Pirfenidone treatment in idiopathic pulmonary fibrosis: nationwide Danish results. Eur Clin Respir J 2016; 3: 32608.

115 Sheikh SI, Hayes D Jr, Kirkby SE, et al. Age-dependent gender disparities in post lung transplant survival among patients with idiopathic pulmonary fibrosis. Ann Thorac Surg 2017; 103: 441-446.

116 Fisher JH, Al-Hejaili F, Kandel S, et al. Multi-dimensional scores to predict mortality in patients with idiopathic pulmonary fibrosis undergoing lung transplantation assessment. Respir Med 2017; 125: 65-71.

117 Song MM, Jones BG, Casanova RA. Auditing sex-and gender-based medicine (SGBM) content in medical school curriculum: a student scholar model. Biol Sex Differ 2016; 7: 93-99.

118 Bismark M, Morris J, Thomas L, et al. Reasons and remedies for under-representation of women in medical leadership roles: a qualitative study from Australia. BMJ Open 2015; 5: e009384.

119 Glauser W. Changes Needed to End “Unconscious Bias' in Hiring at Ontario's Southlake Regional Health Centre, Review Says. 2019. www.theglobeandmail.com/canada/investigations/article-investigation-intosouthlake-hospital-emergency-centre-finds-changes/ Date last accessed: June 2021.

120 McCurry J. Tokyo Medical School Admits Changing Results to Exclude Women. 2018. www.theguardian.com/ world/2018/aug/08/tokyo-medical-school-admits-changing-results-to-exclude-women/ Date last accessed: June 2021.

121 Kawano-Dourado L, Glassberg MK, Molina-Molina M, et al. Gender equity in interstitial lung disease. Lancet Respir Med 2020; 8: 842-843.

122 Coury S, Huang J, Kumar A, et al. McKinsey Report: Women in the Workplace. 2020. www.mckinsey.com/ featured-insights/diversity-and-inclusion/women-in-the-workplace/ Date last accessed: June 2021.

123 Ruzycki SM, Freeman G, Bharwani A, et al. Association of physician characteristics with perceptions and experiences of gender equity in an academic internal medicine department. JAMA Netw Open 2019; 2: e1915165.

124 Association of American Medical Colleges (AAMC). The State of Women in Academic Medicine: 2015-2016. Available from: www.aamc.org/data-reports/faculty-institutions/report/state-women-academic-medicine/ Date last accessed: June 2021.

125 Raviskanthan M, Rees M, Douglass J, et al. Equal or not? Women hold less prestigious roles at respiratory medicine conferences than men. Eur Respir J 2020; 55: 1900701.

126 Vranas KC, Ouyang D, Lin AL, et al. Gender differences in authorship of critical care literature. Am J Respir Crit Care Med 2020; 201: 840-847.

127 Nielsen MW, Bloch CW, Schiebinger L. Making gender diversity work for scientific discovery and innovation. Nat Hum Behav 2018; 2: 726-734.

128 White Hughto JM, Reisner SL, Pachankis JE. Transgender stigma and health: a critical review of stigma determinants, mechanisms, and interventions. Soc Sci Med 2015; 147: 222-231.

129 Baldwin A, Dodge B, Schick VR, et al. Transgender and genderqueer individuals' experiences with health care providers: what's working, what's not, and where do we go from here? J Health Care Poor Underserved 2018; 29: 1300-1318.

130 Hughes M, Pauling JD, Armstrong-James L, et al. Gender-related differences in systemic sclerosis. Autoimmun Rev 2020; 19: 102494. 